\title{
A ECOLOGIA INDUSTRIAL COMO INSTRUMENTO NA BUSCA PELA SUSTENTABILIDADE
}

\author{
INDUSTRIAL ECOLOGY AS A SUSTAINABILITY INSTRUMENT
}

Natiele Dias da Silva- natielepsico2015@gmail.com

André Luiz Oliveira - andre.oliveira@fatectq.edu.br

Faculdade de Tecnologia de Taquaritinga (FATEC) - SP - Brasil

DOI: 10.31510/infa.v15i2.469

\begin{abstract}
RESUMO
A ecologia industrial se apresenta como um importante instrumento na busca pela sustentabilidade, uma vez que tem como princípios não somente a utilização correta e racional de insumos (produção mais limpa, adoção de ecoeficiência, dentre outras ferramentas), mas também a correta gestão dos resíduos sólidos. Utilizando-se como metodologia uma revisão bibliográfica sobre o tema, o objetivo deste estudo foi evidenciar a importância da Ecologia Industrial no contexto empresarial. As empresas que se valem das diretrizes da ecologia industrial em seus processos produtivos experimentam ainda outros benefícios, como a utilização do reconhecimento de "empresa socialmente responsável" como eficaz estratégia de marketing, gerando reflexos das mais diversas ordens, como por exemplo, agregar valor à marca por meio do reconhecimento público oriundo de desempenho financeiro aliado a uma mentalidade produtiva sustentável e responsabilidade social.
\end{abstract}

Palavras-chave: Meio ambiente; Produção Mais Limpa; Desenvolvimento sustentável.

\begin{abstract}
Industrial ecology if presents an important instrument in the search for sustainability, once that has as principles not only the utilizacion correct and rational of inputs (cleaner production, adoption of eco-efficiency, among other tools), but too the correct management of waste solids. Using as a methodology a bibliographic review on the subject, the objective of this study was to highlight the importance of Industrial Ecology in the business context. Companies that if they ar worth of guidelines of industrial ecology in their productives processes experience still other benefits, such as the utilization of the recognition of "socially responsible company" as effective marketing strategy, generating reflexes of the most diverse orders, such for example, to add value the mark by means public recognition, arising of financial performance coupled with a sustainable productive mentality and social responsibility.
\end{abstract}

Keywords: Environment; Cleaner production; Sustainable development. 


\section{INTRODUÇÃO}

Não é recente o entendimento de que a chamada "Responsabilidade Social Empresarial" tornou-se um importante fator de competitividade para os negócios. Há algumas décadas, o que identificava uma organização competitiva era basicamente o preço de seus produtos, mas atualmente outros valores foram agregados a este cenário como por exemplo, a qualidade. Sob esta perspectiva, novas tendências sugerem que as empresas que querem se manter vivas nos próximos anos, devem buscar constante aperfeiçoamento de suas relações com todos os públicos com os quais se relacionam, os chamados "stakeholders.

Para que tais objetivos sejam atingidos, as empresas devem buscar ferramentas que conduzam a este resultado, instrumentos eficazes para que atinjam um nível de excelência não somente em suas finanças, mas também nos aspectos sociais e ambientais. É neste contexto que se apresenta a Ecologia Industrial.

Fabricar produtos ou prestar serviços que não degradem o meio ambiente, promover a inclusão social e participar do desenvolvimento da comunidade de que fazem parte, entre outras iniciativas, são diferenciais cada vez mais importantes para as empresas na conquista de novos consumidores ou clientes. (INSTITUTO ETHOS; SEBRAE, 2003, p. 6)

De acordo com Tachizawa (2006) a gestão ambiental e a responsabilidade social, tornam-se importantes instrumentos gerenciais para capacitação e criação de condições de competitividade para as organizações, qualquer que seja seu segmento econômico.

Segundo Campaner (2011) a necessidade da busca por mudanças e aumento de competitividade propiciou o aparecimento das mais diversas abordagens destinadas a solucionar crises organizacionais, e os novos tempos passariam a caracterizar-se por uma rígida postura dos clientes, voltada à expectativa de interagir com organizações que sejam éticas, com boa imagem institucional no mercado, e que atuem de forma ecologicamente responsável.

Estruturada de forma a resgatar os conceitos desta ferramenta, contextualizando-o dentro da temática sustentabilidade, o presente trabalho busca evidenciar a importância da Ecologia Industrial como uma ferramenta útil e cada vez mais destinada a buscar aspectos relacionados à sustentabilidade nas empresas, por meio de conceitos, técnicas e procedimentos.

Em seguida, foi traçado um breve panorama com relação ao cenário nacional e sua influência na bolsa de valores com a criação de índices oficiais, possibilitando que as 
empresas adaptadas e inseridas nesta realidade utilizem os resultados como estratégia de marketing.

\section{FUNDAMENTAÇÃO TEÓRICA}

Há tempos a sociedade e a academia discutem a impossibilidade de manutenção atual dos padrões de consumo da sociedade capitalista, onde o aumento excessivo de recursos naturais e matérias primas não renováveis, levou à necessidade de modificar a visão de processos de manufatura e o destino dos resíduos sólidos. A demanda produtiva exacerbada requer aumento de produtividade, exigindo, por sua vez, que polos industriais se instalem em grandes centros consumidores, onde a poluição do ar, a destinação incorreta do esgoto e o mau aproveitamento de resíduos sólidos vem se agravando e trazendo grande preocupação à sociedade e aos órgãos de gestão de meio ambiente.

Segundo Almeida e Gianetti (2006) a partir deste contexto social, alternativas são buscadas de forma a combater este panorama. Uma delas diz respeito à chamada "Ecologia Industrial", que pode ser considerada como um sistema produtivo inserido em subsistema produtivo da biosfera, contribuindo para a percepção de que sistemas naturais e produtivos são parte do mesmo sistema.

De acordo com Kravchencko et al. (2016) essa constatação serve para visualizar os processos de manufatura das industrias como ecossistemas industriais sustentados por ecossistemas naturais produtores de matéria prima, priorizando a gestão ambiental e buscando mitigar os impactos ambientais gerados pelo processo produtivo na sociedade em geral.

Um dos conceitos mais importantes da Ecologia Industrial é a identificação com sistemas naturais, delimitando que o sistema produtivo integra sistemas naturais em um mesmo ambiente circundante, não de forma isolada. (BARBIERI, 2016).

Para Fiskel (2009) apud Leitão (2015) a ecologia industrial pode ser definida como uma estrutura para melhorar a eficiência dos sistemas industriais por meio da imitação de aspectos dos ecossistemas naturais, inclusive a transformação cíclica de resíduos em insumos materiais. 


\subsection{Ecologia Industrial em busca da Sustentabilidade}

Segundo Neto et. al (2015) os três pilares básicos que norteiam o desenvolvimento sustentável são: economia, meio ambiente e sociedade, portanto, do ponto de vista industrial todos devem ser estudados sistemicamente. Prossegue o autor afirmando que na indústria de manufatura há a retirada de recursos naturais (input) e através dos processos produtivos ocorre a transformação da matéria prima em produtos finais (output) para comercialização da demanda. No entanto, durante esse percurso há o risco de acidentes ambientais, causando graves danos a comunidade e ao meio ambiente.

De acordo com Trevisan et al. (2016), nota-se uma preocupação das organizações em adotar novos princípios e ferramentas para a produção mais eficiente e com foco na sustentabilidade, pois o meio ambiente funciona com recursos próprios reciclando os outputs de um sistema e transformando em inputs para outros.

A Ecologia Industrial preconiza, portanto, o resíduo "zero" onde o reuso de todas as matérias primas disponíveis se torna indispensável para a cadeia produtiva, assim como ocorre nos ecossistemas naturais. Porém ainda há duvidas de como reutilizar e como armazenar resíduos sintéticos que não são produzidos pela natureza, reconhecidamente perigosos e que por esta razão necessitam de medidas de segurança para proteger a ecosfera de seus efeitos (GIANNETTI et. al 2003).

\subsection{Ecossistema de Produção - uma realidade possível}

Marinho e Kiperstok (2001) relatam que diversos modelos de gestão de processos de manufatura com ênfase na qualidade ambiental foram criados a partir de 1970, uma vez que a preocupação com a escassez de recursos naturais levou especialistas e estudiosos da área a levantarem possibilidades de relação entre os ecossistemas naturais e o ecossistema industrial. Ainda segundo os autores, no ano de 1976 o americano Preston Cloud (Geoquímico) apresentou o primeiro artigo que conteria o termo "Ecossistema Industrial" no Encontro Anual de Geologia Alemã, onde o termo "tecnologia e produção sem resíduos" foi muito discutido.

O artigo "Estratégias de Manufatura" produzido por Robert Frosch e Nicolas Gallopoulos e publicado na revista Scientific American , que é uma referência na consolidação e reconhecimento sobre o tema , alavancaria a utilização de modelos de gestão baseados em processos mais eficientes e menos danosos ao meio ambiente, onde os processos 
isolados são substituídos por sistemas integrados denominados Ecossistemas Industriais (MARINHO e KIPERSTOK, 2001, p. 17).

Segundo Barbieri (2016) os modelos de metabolismo industrial e Ecologia Industrial são modelos de gestão ambiental que visa como fundamento principal aproximar dos sistemas produtivos humanos o que ocorre nos sistemas de decomposição, realizados por micro organismo nos sistemas naturais, onde os organismos extraem alimentos do meio ambiente e devolvem os " restos”, que servirá de alimentos para outros organismos.

$\mathrm{Na}$ atualidade, os processos de recuperação e mitigação de resíduos vivem realidades diferentes de acordo com a matéria prima predominante nos resíduos finais. Materiais tóxicos, inflamáveis e sintéticos são altamente perigosos para a reinserção na cadeia de produção e meio ambiente, sendo necessária a adoção de processos específicos para destinação dos mesmos.

No meio produtivo predominava o entendimento de que os recursos naturais eram ilimitados, assim como a capacidade da natureza de assimilação de resíduos, baseado em um modelo produtivo em que os resíduos são descartados diretamente no meio ambiente com pouco ou nenhum tratamento. Um novo modelo, baseado em teorias contemporâneas e alicerçadas em bases científicas constitui a ideia base da ecologia industrial. ( LEITÃO, 2015) Gama (2010) introduz a ideia da incorporação de tecnologia e informação tendo o " resíduo zero "como resultado principal de suas ações, permitindo ainda o fluxo de energia e materiais entre os componentes do ecossistema . O autor reforça a afirmativa do modelo de processos onde o sistema é aberto quanto ao uso de energia e fechado para o fluxo de materiais.

\section{PROCEDIMENTOS METODOLÓGICOS}

Para a realização desse artigo, foi utilizada uma abordagem qualitativa na busca do entendimento minucioso dos significados e características apresentadas por autores sobre o tema da pesquisa, utilizando-se o método de pesquisa bibliográfica.

\section{RESULTADOS E DISCUSSÃO}

\subsection{Sustentabilidade no Contexto Empresarial brasileiro.}


Para a Comissão Mundial de Meio Ambiente e Desenvolvimento (CMMAD), a definição de Desenvolvimento Sustentável é: "modelo de desenvolvimento que atende as necessidades do presente sem comprometer as gerações futuras a atenderem suas próprias necessidades" (CMMAD, 1991).

De acordo com Souza e Ribeiro (2013) este conceito, aliado ao conceito de desenvolvimento econômico, converge para o tripé do desenvolvimento sustentável, que buscam o fim da pobreza, a redução na poluição ambiental e o desperdício no uso de recursos.

\footnotetext{
"Para reduzir o conflito entre crescimento econômico e proteção ambiental, destaca-se a promoção de uma política de sustentabilidade. A sustentabilidade organizacional tenta conciliar os objetivos tradicionalmente associados com o crescimento econômico e com as limitações ambientais e atividades econômicas" (JUNIOR e GOMES 2010, p.63).
}

Ainda de acordo Gomes Junior e Gomes (2010), a preocupação constante entre Responsabilidade Social e Sustentabilidade Empresarial levou à criação de medidas para avaliar a valorização de empresas que investem em responsabilidade ambiental e social, culminando na criação do chamado índice "Dow Jones Sustainability”, criado em 1999 nos EUA, baseado em um escopo de ações que avaliariam o desempenho financeiro, por empresas que visam a reputação ecologicamente correta no mercado atuante, constituindo-se no primeiro índice a avaliar o desempenho financeiro de empresas ligadas com a mentalidade produtiva sustentável e responsabilidade social.

Para Repetto e Austin (2001) apud Gomes Junior e Gomes (2010) na atualidade o desempenho financeiro dos negócios é significantemente afetado pelos custos e oportunidade apresentado por problemas ambientais, sendo que a combinação de tecnologias ambientais e novas tecnologias de produção, trazem grande potencial competitivo e desempenho superior para empresas envolvidas com ações sustentáveis.

No Brasil, este cenário se repetiu no ano de 2005, quando a BOVESPA (Bolsa de Valores do Estado de São Paulo) criou o chamado "ISE" - Índice de Sustentabilidade Empresarial, com apoio do IFC - International Finance Corporation, braço financeiro do Banco Mundial, cujo desempenho metodológico é coordenado pela Fundação Getúlio Vargas Centro de Estudos de Sustentabilidade. (FGV EAESP, 2017).

A $13^{\circ}$ carteira do ISE, cujo parecer técnico inclui 33 ações de 30 companhias que atendem os critérios de responsabilidade social e desempenho econômico financeiro 
estabelecidos pelo índice, representam 12 setores e a soma de $\mathrm{R}$ 1,28 Trilhões em valores de mercado. (FGV EAESP, 2017).

O Quadro 1 apresenta algumas companhias bem cotadas na carteira da ISE no ano de 2018:

Quadro 1 - Empresas cotadas na Carteira ISE (amostragem).

\begin{tabular}{|l|l|l|l|l|l|}
\hline AES Tiete & CCR & CPFL & Engie & Klabin & Natura \\
\hline B2W & Celesc & Duratex & Fibria & Light & Santander \\
\hline Banco do Brasil & Cemig & Ecorodovias & Fleury & Lojas Americanas & Telefônica \\
\hline Bradesco & Cielo & EDP & Itaú Unibanco & Lojas Renner & Tim \\
\hline Braskem & Copel & Eletropaulo & Itaúsa & MRV & Weg \\
\hline
\end{tabular}

Fonte: BOVESPA 2017

O Instituto de Consultoria e Gestão ETHOS afirma que o papel das empresas com questões ambientais relevantes vai além de melhoria nos sistemas produtivos e busca por insumos que causem menor agressão ao ecossistema, pois as empresas desempenham papel fundamental na capacidade de influenciar o consumidor.

São insustentáveis os padrões de consumo e produção da sociedade atual, e a principal causa dos problemas ambientais, sociais e econômicos. Buscar novos modelos produtivos e padrões de consumo menos agressivos ao meio ambiente é uma tarefa interminável e causara verdadeira revolução no modelo de gestão de recursos ambientais e econômicos mundial, decorre de diversos fatores, principalmente da busca pelo equilíbrio do poder produtivo e a preocupação com os impactos ambientais e sociais (INSTITUTO ETHOS, 2016).

Há uma tendência global de que investidores busquem por empresas socialmente responsáveis, sustentáveis e rentáveis para aplicarem seus recursos, denominados como Investimentos Socialmente Responsável (SRI), pois geram valor a longo prazo e estão mais preparadas para enfrentar os riscos sociais, ambientais e econômicos. (SARTORE, 2012).

Tais índices são formulados com a intenção de criar um modelo de mercado "benchmark", dando aos investidores a oportunidade de identificar as empresas listadas que utilizam práticas sustentáveis de negócios e apresentarem resultados rentáveis (GOMES JUNIOR e GOMES, 2010). 


\subsection{Ecologia industrial e sua relação com o desenvolvimento sustentável.}

Para Eckman, Francis e Ramesh (2005), a Ecologia Industrial formula seus conceitos e propostas segundo a ideia de que "todas as atividade humanas tem uma base material" que deve ser compreendida por todos os setores da sociedade, de forma que a compreensão dessa extensão possa delimitar avanços no progresso industrial, criando novos paradigma de produção e distribuição de bens e serviços efetivamente sustentáveis do ponto de vista social, ambiental, econômico, cultural e político.

Ainda segundo os autores, a desigualdade na distribuição de terras e os sistemas econômico hegemônicos favorecem o crescimento do consumo das classes favorecidas, distanciando se da equidade social e erradicação da pobreza, alavancando a diferença histórica social entre ricos e pobres, de forma que se torna de fundamental importância a participação da sociedade na orientação das mudanças da demanda produtiva atual.

De acordo com dados divulgados pelo PNUMA - Programa das Nações Unidas para o Meio Ambiente, o consumo sustentável é o uso de bens e serviços que atendam às necessidades básicas, proporcionando uma melhor qualidade de vida, minimizando o uso de recursos naturais e materiais tóxicos, a geração de resíduos e a emissão de poluentes durante todo o ciclo de vida do produto ou serviço ( PNUMA, 2014).

\subsection{Atual panorama da ecologia industrial no brasil}

Acredita-se que ainda exista um longo caminho a ser percorrido até que seja atingida a excelência na melhoria contínua dos processos e haja um despertar para a real preocupação com métodos produtivos mais eficientes e limpos. Por outro lado, o setor empresarial vem demonstrando grande interesse no debate sobre a necessidade de mudanças sistêmicas e desenvolvimento de iniciativas setoriais próprias (antecipando iniciativas governamentais), rumo a mudanças mais estruturais como propõe a Ecologia Industrial no cenário brasileiro (LEITÃO,2015).

$\mathrm{Na}$ trilha da busca pela sustentabilidade energética, empresas brasileiras receberam reconhecimento público através do "Prêmio Época Empresa Verde 2015", ao se tornarem industrias $100 \%$ autossuficientes na sua demanda de produção energética. A empresa Honda, por exemplo, investiu na construção de 9 torres de geração de energia eólica para captar ventos, gerando 95 megawatts por ano, evitando assim que 2.200 toneladas de gás carbônico 


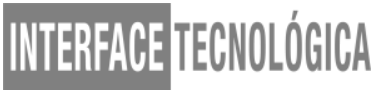

fossem lançadas na atmosfera. O parque supre toda demanda de sua fábrica em Sumaré (Estado de São Paulo) e atende a meta da empresa global em reduzir em 30\% a emissão de gases geradores do efeito estufa. (HERRERO, 2015).

O Brasil foi escolhido para sediar o $10^{\circ}$ Centro Nacional De Tecnologias Limpas (CNTL), evento articulado pela Organização das Nações Unidas Para Desenvolvimento Industrial (UNIDO), onde o Serviço Nacional de Aprendizagem Industrial (SENAI) é responsável por dirigir um dos 74 Centros Nacionais de Produção Mais Limpa espalhados pelo mundo.

O Centro Nacional de Tecnologias Limpas (CNTL) visa facilitar a transferência de informações e tecnologias as empresas, permitindo a incorporação da produção Mais Limpa e outras técnicas de prevenção a poluição em seus sistemas de gestão ambiental, apoiando a sustentabilidade dos negócios. (INSTITUTO SENAI).

A busca pela crescimento econômico de países que tiveram um desenvolvimento industrial tardio como o nosso, e possuem patrimônio ecológico , deve buscar o equilíbrio entre a preservação ambiental e o desenvolvimento econômico , construindo bases de uma políticas econômica viável, e ecologicamente sustentável. (ERCKMAN, FRANCIS e RAMESH, 2005).

\section{CONCLUSÃO}

A tendência relacionada à sustentabilidade empresarial vem ganhando força nos últimos anos à medida em que o setor empresarial compreendeu e internalizou os conceitos de “empresa sustentável e socialmente responsável”, capaz de lidar de forma eficaz com os riscos econômicos, ambientais e sociais, gerando valor para seus acionistas.

A Ecologia Industrial mostra-se um aliado importante não somente com relação à adoção de medidas que conduzam a uma produção sustentável, mas também uma mudança de mentalidade de todos os colaboradores e stakeholders.

Este cenário se reflete na criação do ISE, uma iniciativa pioneira que tem por objetivo promover o estímulo à responsabilidade ética das corporações, sem perder de vista os critérios de eficiência econômica que norteiam o mundo empresarial, além da busca pelo equilíbrio ambiental, justiça social e governança corporativa.

Não obstante os benefícios diretos de gestão identificados com a adoção da ideologia baseada na Ecologia Industrial, as empresas obtém benefícios indiretos de extrema relevância, 


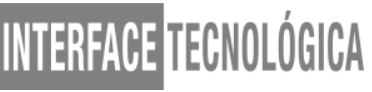

tal como a possibilidade de explorar como estratégia de marketing a boa imagem decorrente de uma organização socialmente responsável, agregando valores intangíveis ao seu patrimônio corporativo.

\section{REFERÊNCIAS}

ALMEIDA, C. M. V. B. de; GIANNETTI, B. F. Ecologia industrial: conceitos, ferramentas e aplicações. São Paulo: Edgard Blucher, 2006 p.31.

BOVESPA . BRASIL BOLSA BALCÃO. B3 divulga a $13^{\circ}$ carteira do ISE -Indice de Sustentabilidade Empresarial, BM\&FBOVESPA. Mercado Financeiro - Publicações 2018. Disponível em :< https://www.isebvmf.com.br/b3-divulga-a-13-carteira-do-ise-indice-desustentabilidade-empresarial/?locale=pt-br $>$ Acesso em 10/ago/2018

BARBIERI, J. C. Gestão Ambiental Empresarial: Conceitos, Modelos e Instrumentos. 4. ed. São Paulo: Saraiva, 2016 .

CAMPANER, E.C.S \& SILVA, H.H. R. Gestão Ambiental e Responsabilidade Social: Uma Questão Passageira? Universidade Unisalesiano .III Encontro Científico e Simpósio de Educação UNISALESIANO 2011.

CMMAD - Comissão Mundial sobre Meio Ambiente e Desenvolvimento. Nosso futuro comum. 2a ed. Tradução de Our common future. 1a ed. 1988. Rio de Janeiro : Editora da Fundação Getúlio Vargas, 1991.

ERCKMAN,S; FRANCIS,C; RAMESH,R . Ecologia Industrial: Uma Agenda para a Evolução do Sistema Industrial. São Paulo . Instituto Polis .2005.88p.( Caderno para Proposições do Século XXI). Disponível em: http://www.bibliotecadigital.abong.org.br/bitstream/handle/11465/390/POLIS_ecologia_indus trial_uma_agenda_para_educa\%E7\%E3o.pdf?sequence=1 Acesso em 30/ago/2018.

FGV-EAESP . ISE - Indíce Sustentabilidade Empresarial . O que é ISE ? Centro de Estudo em Sustentabilidade, Fundação Getúlio Vargas FGV - EAESP 2017. Disponível em : https://www.isebvmf.com.br/o-que-e-o-ise?locale=pt-br Acesso em : 21/jul/2018.

GAMA , M. Blog Sustentabilidade em Ação Marcio Ambiental . Ecossistemas Industriais Evolução Sistemas Produtivos . Brasil , Março 2010. Disponível em : https://marcioambiental.wordpress.com/2010/03/17/ecossistemas-industriais-evolucao-desistemas-produtivos/ > Acesso em 01/ago/2018

GIANETTI, F.B et al. Implantação de Eco - Tecnologias rumo à Ecologia Industrial. Gestão da Produção. Scielo. RAE Eletrônica . vol. 2, nº.1 ,São Paulo - June 2003. Disponível em : $<$ http://www.scielo.br/scielo.php?script=sci_arttext\&pid=S167656482003000100011\&lang=pt > Acesso em 20/ago/2018. 
GOMES,J S.F ; GOMES,A.R . As Vantagens da Sustentabilidade Empresarial . Revista INGEPRO - Inovação , Gestão e Produção. Vol.02 , n. ${ }^{\circ} 6$. Agosto de 2010. Disponível em $:<$ http://ingepro.com.br/Publ_2010/Agost/286-787-1-PB.pdf> Acesso em 15/jul/2018

HERRERO,T. Blog do Planeta . Prêmio Época Empresa Verde: As Empresas Responsáveis com o Planeta 2015. Revista Època 2015 . Disponível em: https://epoca.globo.com/colunase-blogs/blog-do-planeta/noticia/2015/10/empresas-responsaveis-com-o-planeta-de-2015.html Acesso em : 20/agos/2018

INSTITUTO ETHOS de Empresas e Responsabilidade Social . Incentivo a Gestão Empresarial Socialmente Responsável . Organização - Meio Ambiente .São Paulo.Disponível em $\quad<\quad$ https://www3.ethos.org.br/conteudo/gestao-socialmenteresponsavel/meio-ambiente/\#.W6DuWs5KjIX> Acesso em : 09/ago/2018 .

INSTITUTO ETHOS de Empresas e Responsabilidade Social. Perguntas freqüentes. São Paulo, 2009. Disponível em: < http://www.ethos.org.br/DesktopDefault.aspx?TabID=3344\&Alias=Ethos\&Lang= > Acesso em: $10 / j u l / 2018$

KRAVCHENKO,G.A; PASQUALETO, A ; FERREIRA, E.M. Aplicação de Princípios da Ecologia Industrial nas Empresas Moveleiras. Artigo Técnico - Scielo .Engenheira . Sanitária Ambiental, vol.21, $\mathrm{n}^{\circ}$ 2, Rio de Janeiro , Abril/Junho 2016. Disponível em : http://www.scielo.br/scielo.php?script=sci_arttext\&pid=S1413-41522016000200283 Acesso em : 23/ago/2018

LEITÃO, A . Economia Circular : Uma Nova Filosofia de Gestão Para O Século XX. Portuguese Journal of Finance, Management and Accouting. PJFMA vol.1, N²,Setembro 2015.Disponível<http://www.u3isjournal.isvouga.pt>Acesso em: 15/jul/2018

MARINHO, M; KIPERSTOK, A. Ecologia Industrial e Prevenção da Poluição : Uma contribuição ao debate regional . Bahia Análise \& Dados - Salvador .BA, SEI vol.10, nº 271.279, Março 2001. Disponível em : http://teclim.ufba.br/site/material_online/publicacoes/pub_art10.pdf Acesso em : $15 /$ junh/2018

NETO, G.C.O; GODINHO, M.F; GANGA, G.M.V; NAAS, I.A; VENDRAMETTO,O. Princípios e Ferramentas da Produção Mais Limpa : Um Estudo Exploratório em Empresas Brasileiras .Scielo RAE Eletrônica . Gestão da Produção, vol.2,n .2, São Carlos . June 2015, Disponível em< http://www.scielo.br/pdf/gp/v22n2/0104-530X-gp-22-2-326.pdf> Acesso em $: 15 / j u l / 2018$

SARTORE, M.S . O Mercado Socialmente Responsável . Universidade Estado de São Paulo. FCLAR REDD. Revista Espaço Diálogo e Desconexão . Araraquara, v.4, n², Jan/Jul. 2012. Disponível em : 
<https://periodicos.fclar.unesp.br/redd/article/download/5185/4250>

Acesso em : $18 / \mathrm{jul} / 2018$

PNUMA. Programa das Nações Unidas para o Meio Ambiente . PNUMA no Brasil. 2014. Disponível em:<http.www.pnuma.org.br/interna.php/id=44>. Acesso em: 01/set/2018

SENAI - Instituto Inovação e Tecnologia . SENAI /RS Instituto de Tecnologia Couro e Meio Ambiente. Centro Nacional de Tecnologias Limpas (CNTL) . Departamento Regional Porto Alegre - Brasil. Disponível em : http://institutossenai.org.br/conteudo/cntl Acesso em: 09/ago/2018.

SOUZA, M.T.S; RIBEIRO,H.C.M; Sustentabilidade Ambiental: Uma meta -Análise da Produção Brasileira em Periódicos de Administração. Revista Administração Contemporânea .Scielo . ANPAD - RAC, Rio de Janeiro, vol.17,n³, art 6,p.p 368-396 Maio/Junho 2013. Disponível em : http://www.scielo.br/scielo.php?script=sci_arttext\&pid=S1415-65552013000300007 Acesso em : $11 / j u l / 2018$

TACHIZAWA, T. Gestão ambiental e responsabilidade social corporativa. 4. ed. São Paulo: Atlas, 2006.

TREVISAN, M. et al. Ecologia Industrial , Simbiose Industrial e Ecoparque Industrial: Conhecer para Aplicar. Revista Eletronica Sistema \& Gestão. V.11, n² . Ano 2016. Disponível em : < http://www.revistasg.uff.br/index.php/sg/article/view/993/430> Acesso em : 01/jul/2018 